\title{
Perfil químico e atividades antioxidante e antifúngica do óleo essencial da flor de Cochlospermum regium (Mart. ex Schrank.) -Pilg. (Bixaceae)
}

Antonio Carlos Pereira de Menezes Filho ${ }^{1}$, Wendel Cruvinel de Sousa ${ }^{1}$, Marcela Christofoli ${ }^{2}$, Carlos Frederico de Souza Castro ${ }^{1}$

${ }^{1}$ Instituto Federal Goiano - IF Goiano, Campus de Rio Verde, GO. ${ }^{2}$ Universidade Federal de Goiá - UFG, Campus Samambaia, Goiânia, GO. E-mail: astronomoamadorgoias@gmail.com

\section{Resumo}

O domínio Cerrado apresenta inúmeras espécies vegetais que produzem naturalmente óleos essenciais que são incluídos em uma das várias classes de metabólitos secundários. Objetivou-se caracterizar a constituição química e avaliar as atividades antioxidante e antifúngica do óleo essencial das flores de Cochlospermum regium. As flores de C. regium foram coletadas em área de Cerrado, em Rio Verde/GO, Brasil. Para extração do óleo essencial, foram utilizadas $100 \mathrm{~g}$ de flores, e em seguida, foram submetidas à hidrodestilação em aparelho tipo Clevenger, e o rendimento de óleo essencial, determinado em porcentagem. Em seguida, o óleo essencial foi analisado quanto à composição por cromatografia gasosa com espectrometria de massas acoplada CG-EM, e determinado quanto ao potencial antioxidante na redução do DPPH e como antifúngico por difusão em ágar sobre o isolado de Sclerotinia sclerotiorum. 0 rendimento médio de óleo essencial foi igual a 0,10\%. Foram identificados 32 compostos, destacando-se os majoritários cariofileno E 9,76\%, $\gamma$-muuroleno $16,69 \%$ e biciclogermacreno 39,82\%. A atividade antioxidante apresentou $100 \%$ de redução do radical DPPH, para as concentrações entre 50 a $2 \mathrm{mg} \mathrm{mL}^{-1}$, e reduções de 85,77 a $13,18 \%$ ( $E_{50}$ ) para as concentrações 1 a $0,031 \mathrm{mg} \mathrm{mL}^{-1}$ de óleo essencial. Conclui-se que o óleo essencial de $C$. regium apresentou baixo rendimento, no entanto, contém muitos compostos sesquiterpenos hidrocarbonados e oxigenados, apresentando-se como excepcional agente antioxidante e antifúngico natural no combate a Sclerotinia sclerotiorum. Sendo assim, o óleo essencial das flores de $C$. regium se apresenta como um forte candidato para novas pesquisas que poderão ser aplicadas nas áreas da saúde, agrícola e alimentícia.

Palavras chaves: Cochlospermum regium; DPPH; hidrodestilação; óleo essencial.

Chemical profile and antioxidant and antifungal activites of the essential oil from Cochlospermum regium (Mart. ex Schrank.) -Pilg. (Bixaceae) flowers

\begin{abstract}
The Cerrado domain has numerous plant species that naturally produce essential oils, that are include in one of several classes of secondary metabolites. The objective of this study was to characterize the chemical composition and to evaluate the antioxidant and antifungal activities of the essential oil from Cochlospermum regium flowers. The flowers from C. regium were collected in an area of Cerrado, in Rio Verde/GO, Brazil. To extract the essential oil, $100 \mathrm{~g}$ of flowers were used, and then they were subjected to hydrodistillation in Clevenger-type apparatus, and the essential oil yield, determined in percentage. Then, the essential oil was analyzed for composition by gas chromatography with coupled mass spectrometry GCMS, and determined as to the antioxidant potential in reducing DPPH and, as antifungal by agar diffusion in the isolate from Sclerotinia sclerotiorum. The essential oil was analyzed by gas chromatograph associated with mass spectrometry CG-MS and its antioxidant potential was evaluated by the free radical sequestration technique DPPH. The average yield of essential oil was $0.10 \% .32$ compounds were identified, in especially majority caryophyllene E $9.76 \%$, $y$-muurolene $16.69 \%$ and bicyclogermacrene $39.82 \%$. The antioxidant activity showed $100 \%$ reduction in the DPPH radical, for concentrations between 50 to $2 \mathrm{mg}$ $\mathrm{mL}^{-1}$, and reductions of 85.77 to $13.18 \%\left(\mathrm{IC}_{50}\right)$ for concentrations 1 to $0.031 \mathrm{mg} \mathrm{mL}^{-1}$ of the essential oil. It is
\end{abstract}


concluded that the essential oil from C. regium showed low yield, however, it contains many hydrocarbon and oxygenated sesquiterpenes compounds, presenting itself as an exceptional natural antioxidant and antifungal agent in the fight against Sclerotinia sclerotiorum. Thus, the essential oil of the flowers from $C$. regium presents itself as strong candidate for new research that can be applied in the areas from health, agricultural and foods.

Keywords: Cochlospermum regium; DPPH; hydrodistillation; essential oil.

\section{Introdução}

O domínio Cerrado é o segundo maior em área territorial, espécies da flora e fauna brasileira, ocupando $23 \%$ do território nacional, sendo considerado um domínio de transição entre os mais variados biomas brasileiros (MENEZES FILHO et al., 2020a; BATALHA, 2011; NETO; MORAIS, 2003). Conforme Beuchle et al. (2015), e Mendonça et al. (1998), o Cerrado apresenta cerca de 7.000 táxons vegetais, sendo que, cerca de 6.682 são nativos, estando distribuídos em 170 famílias e mais de 1.140 gêneros. A cada ano, novas espécies vegetais são classificadas neste ambiente. Dentre estas inúmeras espécies vegetais, várias são estudadas partindo do princípio que são produtoras de metabólitos secundários de grande importância farmacológica e bioativa, sendo encontrados em diferentes órgãos vegetativos, desde o rizoma até as sementes (BARBOSA et al., 2017; ANDRADE et al., 2012; CAMILLO et al., 2009).

O algodão-do-cerrado ou algodãozinho do cerrado (Cochlospermum regium) é uma das espécies vegetais que possuem inúmeras classes de metabólitos secundários, dentre elas, os óleos essenciais que estão distribuídos em várias partes dos órgãos vegetativos (terrestre e aéreo). Esta espécie pertence atualmente a Família Bixaceae, sendo encontrado em ambientes de Cerrado sensu stricto, rupestre, ralo, típico, cerradão e em áreas antropomorfizadas (MENEZES FILHO; CASTRO, 2020; BATISTA et al., 2014; SÓLON et al., 2012). Esta espécie vegetal apresenta-se como um subarbusto com até $2 \mathrm{~m}$ de altura, os ramos apresentam tamanho médio entre 0,8 a 1,8 $\mathrm{m}$ de comprimento, o rizoma é robusto e do tipo lenhoso, as folhas são alternadas, simples, longopecioladas e palmatificadas. As flores apresentam forma de concha, entre 5 a 10 flores, com tamanho variando entre 6 a $8 \mathrm{~cm}$ de diâmetro. 0 fruto apresenta forma de cápsula deiscente, ovoide ou elipsoide, com cerca de $6 \mathrm{~cm}$ de comprimento, apresentando internamente sementes envoltas em filamentos lanosos (felpado) (CAMILLO et al., 2016).

Pouco se conhece sobre a constituição química volátil de $C$. regium, e ainda inexistem trabalhos que avaliam a atividade antioxidante e antifúngica do óleo essencial de suas flores. Brum et al. (1997), estudaram o óleo essencial extraído do rizoma o qual apresentou importante atividade antimicrobiana. Já Rouquayrol et al. (1980), avaliando o óleo essencial extraído das folhas sobre uma possível ação moluscicida, não observaram tal efeito de inibição avaliado. Um grande número de trabalhos avaliam algumas ações como anti-inflamatória, analgésica, antibacteriana e atividade antioxidante utilizando extratos do rizoma de $C$. regium que já é bem caracterizado (SÓLON et al., 2009; ANDRADE et al., 2008).

Os compostos voláteis são produzidos naturalmente pelas plantas como resposta fisiológica a diferentes tipos de estresses ambientais (radiação solar, índice pluviométrico, ataque por insetos, herbivoria, altitude da área e nutrientes do solo) e genéticos entre populações de indivíduos, sendo caracterizados como metabólitos secundários. Esses metabólitos podem sofrer variação por fatores intrínsecos ou extrínsecos (SILVA et al., 2019c; BATISTA et al., 2019; SOUSA; SOUSA, 2017).

Estes fatores ambientais e genéticos podem influenciar na atividade antioxidante $\mathrm{e}$ antifúngica dos óleos essenciais, os quais tem ganhado espaço e atenção na comunidade científica devido as suas propriedades anti deletérias causadas pelos radicais livres, que ocasionam diariamente inúmeros casos de cânceres, cirrose, arterosclerose e artrite correlacionados com o stress oxidativo, bem como podem proporcionar diferentes níveis de potencialidade moduladora sobre uma cepa de microrganismo patogênico (SILVA et al., 2019a; GASTALDI et al., 2016; MORAIS et al., 2006). Além disso, inúmeros óleos essenciais apresentam variadas ações biológicas como, inseticida, 
larvicida, moluscicida, nematicida, tripanossomida, citotoxicidade, antiprotozoária, bem como, na preservação de frutas pós-colheita e na alelopatia (CALDAS et al., 2020; SILVA et al., 2019b; BEZERRA et al., 2019; COSTA et al., 2016; MARCO et al., 2015).

O uso de óleos essenciais como potenciais agentes fungicidas biológicos, possibilita o controle de fitopatógenos, como o Sclerotinia sclerotiorum, causador do mofo branco. Esse fitopatógeno ocasiona grandes perdas na produtividade anual, principalmente na cultura de soja causando a podridão branca da haste (SILVA et al., 2018a; GARCIA et al., 2012). As hastes de soja doentes apresentam lesões moles, de coloração acastanhada, com micélio branco, cobrindo as partes do tecido lesionado. 0 fungo $S$. sclerotiorum é de difícil controle em ambiente natural, uma vez que produz estruturas de resistência conhecida por escleródios, que podem permanecer viável no solo por até 11 anos (MENEZES FILHO et al., 2020a; HAMID et al., 2018; SILVA et al., 2018a; JULIATTI et al., 2013; ).

A maioria dos fungicidas químicos registrados é utilizada de forma indiscriminada ocasionando sérios problemas ambientais como contaminação da água, solos, fauna e para a saúde humana (BOMFIM et al., 2015). Com isso, a necessidade de se produzir novos agentes fungicidas com princípios químicos naturais, vem ganhando interesse nas últimas décadas, devido a fácil aquisição, baixo custo e ausência de problemas inerentes aos produtos químicos (KUMAR et al., 2014).

Sendo assim, o trabalho teve por objetivo avaliar o perfil químico, e as atividades antioxidante e antifúngica presentes no óleo essencial das flores de Cochlospermum regium sobre o fungo Sclerotinia sclerotiorum.

\section{Material e Métodos \\ Coleta do material botânico}

As flores de $C$. regium foram coletadas no mês de outubro de 2018 em área de Cerrado variante (Cerrado denso a ralo), localizado na Universidade de Rio Verde/GO, Brasil, com a seguinte coordenada geográfica: $17^{\circ} 47^{\prime} 13.0^{\prime \prime} \mathrm{S}$ $50^{\circ} 57^{\prime} 58.9^{\prime \prime} \mathrm{W}$ (GPS Garmin, Mod. GPSMAP $64 \mathrm{X}$ (Glonass). O material vegetal foi coletado nas primeiras horas do dia, entre as 6 e $8 \mathrm{~h}$ da manhã, sendo armazenadas em sacos plásticos de cor preta. O material foi encaminhado para o laboratório de Química Tecnológica, para procedimento de extração. Uma exsicata foi identificada, herborizada e depositada no Herbário do Instituto Federal Goiano com Voucher HRV 844.

\section{Extração do óleo essencial}

Para extração e rendimento do óleo essencial, as flores tiveram sua massa em triplicata determinada, sendo que para cada amostra pesou-se cerca de $100 \mathrm{~g}$ de flores. Em seguida, as flores foram processadas em processador doméstico (Philips Walita, Mod. Viva Collection) com $500 \mathrm{~mL}$ de água destilada e deionizada. 0 processo de extração foi realizado em aparato tipo Clevenger (Marconi, Mod. MA553/2000) por 4 horas sob refluxo. O hidrolato foi separado com três lavagens utilizando $30 \mathrm{~mL}$ de diclorometano (Alphatec, P.A - ACS, pureza 100\%). Em seguida, as frações foram reunidas e acrescentou-se sulfato de sódio anidro (Fmaia, P.A - ACS, pureza 99,8\%) e foi feita a filtragem em papel de filtro qualitativo faixa azul (Unifil, C42). O rendimento expresso em (\%) de óleo essencial ( $\mathrm{m} / \mathrm{v}$ ) foi determinado conforme equação 1 pela diferença de massa.

$\%$ Rendimento $=$ (óleo extraído/peso massa fresca vegetal) $\times 100$

Eq. (1).

\section{Análise por CG-EM}

A análise dos constituintes químicos do óleo essencial das flores de $C$. regium foi realizada em sistema de cromatógrafo a gás acoplado ao espectrômetro de massas sequencial (CG-EM), equipado com auto-injetor (Combi PAL AOC-5000 Shimadzu), coluna Restek Rtx-5ms (30m x 0,250 mm x 0,25 $\mu \mathrm{m}$ ) fundida com sílica, e espectrômetro de massas sequencial (MSTQ8030 Shimadzu), com por ionização por impacto eletrônico (IE) (70 e.V). A temperatura inicial foi mantida a $60{ }^{\circ} \mathrm{C}$ por 3,0 min., seguido de um acréscimo de $3{ }^{\circ} \mathrm{C} \min ^{-1}$ até atingir $200{ }^{\circ} \mathrm{C}$ e posteriormente foi programada para um aumento de temperatura de $15{ }^{\circ} \mathrm{C} \mathrm{min}$ até 280 ${ }^{\circ} \mathrm{C}$, permanecendo nessa temperatura por mais 1,0 min.

As temperaturas do injetor e do detector foram de $230{ }^{\circ} \mathrm{C}$ e $300{ }^{\circ} \mathrm{C}$, respectivamente. As análises foram realizadas utilizando gás He como carreador, e pressão de injeção de $57,4 \mathrm{KPa}$, razão de Splitless: 150, faixa de detecção do espectrômetro de massas: $43-550 \mathrm{~m} / \mathrm{z}$, start time (cut time do solvente): 3,0 min e fluxo de $3 \mathrm{~mL}$ $\min ^{-1}$. A identificação dos componentes do óleo foi baseada no índice de retenção linear (índice de Kovats) (IK) calculado em relação aos tempos 
de retenção da série homóloga de $n$-alcanos (C07 a (-40) e no padrão de fragmentação observado nos espectros de massas, por comparação destes com a literatura (ADAMS, 2007) e da espectroteca (Nist 11).

\section{Atividade antioxidante por DPPH}

O óleo essencial de $C$. regium foi avaliado quanto à capacidade antioxidante, pelo método de sequestro do radical DPPH (2,2-difenil-1picrilhidrazil) (Sigma-Aldrich). O método por DPPH seguiu conforme Mezza et al. (2018) com modificações, para uso em microplaca (Videplast) de 96 poços em microdiluições. Para cada poço foram adicionados $100 \mu \mathrm{L}$ de uma solução metanólica de DPPH 0,06 mMol e $100 \mu \mathrm{L}$ de uma solução metanólica de óleo essencial em diferentes concentrações decrescentes (50; 40; 30 ; 20; 10; 5,$0 ; 2,5 ; 1,25 ; 1,0 ; 8,0 ; 6,0 ; 4,0 ; 2,0$; 1,$0 ; 0,5 ; 0,25 ; 0,125 ; 0,062$ e $\left.0,031 \mathrm{mg} \mathrm{mL}^{-1}\right)$.

A microplaca foi mantida em repouso em local ao abrigo da luz e calor por 2 horas, logo em seguida, foram realizadas as leituras em espectrofotômetro de microplacas (Polaris, Mod. MA 616) em $517 \mathrm{~nm}$. A capacidade porcentual de sequestro de radical livre DPPH foi calculada conforme equação 2 .

$\begin{array}{lllll}\left(\left(\begin{array}{lll}\mathrm{AC} & - & \mathrm{AS}\end{array}\right) / \mathrm{AC}\right) & \mathrm{x} & 100\end{array}$

Eq. (2)

Onde: AC, absorção da solução controle e AS absorções das soluções de óleos essenciais contendo o radical DPPH.

\section{Atividade antifúngica em difusão em ágar}

$\mathrm{O}$ isolado de $\mathrm{S}$. sclerotiorum foi obtido a partir de escleródios formados no interior da haste de soja, proveniente de campos comerciais no município de Rio Verde-GO, Brasil. O meio de cultura batata, dextrose e ágar (BDA) (Kasvi) foi doado pelo laboratório de Química de Produtos Naturais do IF Goiano, Campus Rio Verde-GO, Brasil.

A atividade antifúngica dos óleos essenciais de $C$. regium sobre o crescimento micelial de $S$. sclerotiorum foi avaliada através de diferentes concentrações, partindo de 100 (óleo puro); 50; 25 ; 12,$5 ; 6,25 ; 3,125$ e $1,5625 \mu \mathrm{L} \mathrm{mL}^{-1}$ de óleo essencial. Como controle negativo, utilizou-se a testemunha (ausência de óleo essencial) e dimetilsulfóxido (DMSO) (Vetec, P.A ACS, pureza $100 \%$ ), e como controle positivo fungicida de referência Frowncide 500 SC, na concentração de $10 \mu \mathrm{LL}^{-1}$.
As concentrações do óleo essencial foram adicionadas ao meio de cultura BDA após esterilização e resfriamento, bem como para os tratamentos com fungicida comercial e DMSO. Após solidificação do meio, em câmara de fluxo laminar vertical (Marconi, Mod. 100 MA 1550I), 1 disco de micélio de $S$. sclerotiorum com $7 \mathrm{~mm}$ de diâmetro, foi depositado no centro da placa de Petri de $9 \mathrm{~cm}$ de diâmetro. Em seguida foram incubadas (Nova Instruments, Mod. NI 1523i) à temperatura de $20{ }^{\circ} \mathrm{C}$, conforme descrito por Garcia et al. (2012), com adaptações.

A avaliação consistiu em medições diárias do diâmetro das colônias com paquímetro digital (Digimess, Mod. 100.174BL) resolução 150 $\mathrm{mm} / 6^{\prime \prime}$, iniciadas após 24 horas do início da incubação e encerradas 48 horas após, quando as colônias fúngicas, do tratamento testemunha, atingiram completamente a área interna da placa. A determinação do percentual de inibição de crescimento micelial (PIC), foi realizada conforme equação 3, proposto por Garcia et al. (2012).

$\mathrm{PCl}=(\mathrm{DTT}-\mathrm{DTQ}) / \mathrm{DTT} \times 100$ Eq. (3)

Onde: $\mathrm{PCl}=$ percentual de inibição de crescimento, DTT = diâmetro no tratamento testemunha, $\mathrm{DTQ}=$ diâmetro no tratamento químico.

\section{Análise estatística}

A determinação do rendimento de extração do óleo essencial e atividade antioxidante foram realizadas em triplicata seguidas de \pm desvio padrão. Os dados foram submetidos à análise de Variância (ANOVA), e para determinação da diferença significativa, foi utilizado teste de Tukey a $(p \leq 0,05)$. O delineamento experimental aplicado ao teste antifúngico, consistiu em um sistema inteiramente casualizado em esquema fatorial com 7 (concentrações de óleo essencial) +3 (controles negativo e positivo), com 4 repetições. Os dados foram avaliados a partir da análise de Variância pelo teste $F$ a $1 \%$ de significância e Tukey a $5 \%$ de significância. O software estatístico utilizado foi o PAST 3 (versão livre, $3.22,2018)$.

\section{Resultados e Discussão}

$O$ rendimento do óleo essencial das flores de C. regium foi igual a $0,10 \%(\mathrm{~m} / \mathrm{v}) \mathrm{com}$ coeficiente de variação (CV) de 0,30. Comparando com outros gêneros vegetais, 
devido à falta de trabalhos, Menezes Filho (2020b), obteve rendimento de $0,01 \%$ para o óleo essencial extraído das flores de Fridericia platyphylla. Nascimento et al. (2018), obtiveram rendimento de extração de $0,2 \%$ para o óleo essencial das flores de Xylopia aromatica. Já no estudo realizado por Souhaiel et al. (2017), avaliando o rendimento do óleo essencial das flores de Ammoides pusilla, os pesquisadores obtiveram rendimento de $1,6 \%$.

O rendimento do óleo essencial das flores de $C$. regium é considerado baixo quando comparados a outros estudos avaliando o rendimento de óleo essencial de outras espécies em vários grupos botânicos. Entretanto, projetos de conservação de $C$. regium devem ser realizados, garantindo a perpetuação da espécie, podendo assim, ser realizado processo de extrativismo vegetal consciente e ecologicamente correto, o mesmo é discutido por Carneiro et al. (2016) e Sila et al. (2015).

Foram observados 3 compostos majoritários no óleo essencial das flores de Cochlospermum regium, cariofileno E com 9,76\%, $\psi$-muuroleno $16,69 \%$ e biciclogermacreno $39,82 \%$, com total de $81,13 \%$ de compostos sesquiterpenos hidrocarbonados e de 15,67\% para sesquiterpenos oxigenados (Tabela 1).

$\mathrm{Na}$ Tabela 1 está apresentado o perfil químico do óleo essencial das flores de $C$. regium por CG-EM coletadas em área de Cerrado no município de Rio Verde, Goiás, Brasil.

A comparação entre a composição química do óleo essencial floral devido a falta de trabalhos para $C$. regium, foi comparada entre outros grupos botânicos, como para os estudos de Nascimento et al. (2018), onde encontraram 28 compostos químicos no óleo essencial das flores de Xylopia aromatica, sendo o biciclogermacreno com 9,74\%, zonareno $2,90 \%$, $\delta$-cadieno $\quad 2,40 \%, \quad 7$-Epi- $\alpha$-eudesmol $\quad 7,76 \%$, khusinol $7,23 \%$ e cedro-8(15)-em-9- $\alpha$-ol com 4,40\%. Estudo desenvolvido por Cerqueira et al. (2009), avaliaram os componentes do óleo essencial das flores de Myrcia salzmannii coletadas em diferentes períodos do ano, onde os compostos com maior concentração observados foram $\beta$-cariofileno com $13,8 \%$ e $\alpha$ humuleno com $10,9 \%$.

O óleo essencial das flores de Prangos ferulacea analisado por Bazdar et al. (2018), apresentou como compostos majoritários o $\alpha$ pineno com $20,91 \%$ e bornil acetato com $13,80 \%$. Já Juiz et al. (2016), encontraram para os óleos essenciais das flores de Ocimum americanum e Ocimum basilicum os seguintes compostos majoritários, E-cinamato de metila com $54,4 \%$, metil chavicol 6,8\%, 1,8-cineol 6,7\% e linalol 5,6\% (O. americanum), e metileugenol com $18,0 \%$, elimicina $14,6 \%$, cariofileno $12,0 \%$ e 1,8-cineol com $11,2 \%$ (O. basilicum).

Ressaltando o estudo realizado por Brum et al. (1997) avaliando os constituintes químicos do óleo essencial do rizoma de Cochlospermum regium por CG-EM, encontraram os seguintes compostos, $\beta$-selineno com $34,1 \%$, elemeno 5,4\%, Trans-cariofileno 4,8\%, $\alpha$-pineno 3,4\%, $\alpha$ humuleno 2,8\%, aromadendreno 2,1\%, $\alpha$ selineno $1,2 \%$ e $\delta$-cadineno com $0,8 \%$. 
Tabela 1. Perfil químico do óleo essencial das flores de Cochlospermum regium por CG-EM.

\begin{tabular}{|c|c|c|c|c|}
\hline Compostos & RT (min) & $\mathbf{R} \mathbf{I}_{\text {exp }}$ & $\mathbf{R} \mathbf{l}_{\text {lit }}$ & AR\% \\
\hline Mirceno & 4,083 & 994 & 988 & 0,46 \\
\hline$O$-cimeno $<(E)-\beta->$ & 4,708 & 1047 & 1044 & 0,21 \\
\hline$\nu$-terpineno & 4,914 & 1064 & 1054 & 0,13 \\
\hline Terpinen-4-ol & 6,478 & 1184 & 1174 & 0,46 \\
\hline$\alpha$-terpineol & 6,634 & 1195 & 1186 & 0,57 \\
\hline$\delta$-elemeno & 8,594 & 1336 & 1335 & 0,81 \\
\hline$\alpha$-ilangeno & 9,150 & 1376 & 1373 & 0,67 \\
\hline Sativo & 9,308 & 1388 & 1390 & 1,21 \\
\hline Cariofileno E & 9,783 & 1423 & 1417 & 9,76 \\
\hline$\beta$-gurjuneno & 9,872 & 1429 & 1431 & 0,12 \\
\hline$\beta$-copaeno & 10,010 & 1440 & 1430 & 1,23 \\
\hline Aromadendreno & 10,110 & 1447 & 1439 & 0,88 \\
\hline$\alpha$-humuleno & 10,212 & 1455 & 1452 & 3,16 \\
\hline Aromadendreno $<$ Allo- $>$ & 10,310 & 1462 & 1460 & 0,38 \\
\hline v-muuroleno & 10,553 & 1481 & 1478 & 16,69 \\
\hline$\beta$-selineno & 10,633 & 1487 & 1489 & 1,78 \\
\hline Biciclogermacreno & 10,768 & 1497 & 1500 & 39,82 \\
\hline$\alpha$-fameseno $\mathrm{E}$ & 10,851 & 1503 & 1505 & 0,28 \\
\hline Y-cadieno & 10,919 & 1508 & 1513 & 0,18 \\
\hline$\delta$-amorfeno & 10,988 & 1514 & 1511 & 2,36 \\
\hline Bisaboleno $<(E)$-Iso- $\gamma->$ & 11,034 & 1517 & 1528 & 1,05 \\
\hline$\delta$-cadieno & 11,197 & 1530 & 1522 & 0,15 \\
\hline Nerolidol Z & 11,328 & 1540 & 1531 & 0,43 \\
\hline Dauca-4-(11), 7-diene<Trans-> & 11,523 & 1556 & 1556 & 0,55 \\
\hline Palustrol & 11,648 & 1566 & 1567 & 0,93 \\
\hline Spatulenol & 11,749 & 1574 & 1577 & 1,72 \\
\hline Óxido de Cariofileno & 11,843 & 1581 & 1582 & 3,83 \\
\hline Globulol & 11,945 & 1589 & 1590 & 2,97 \\
\hline Rosifoliol & 12,031 & 1596 & 1600 & 0,54 \\
\hline Eudesmol $<5-E p i-\alpha->$ & 12,272 & 1615 & 1607 & 1,08 \\
\hline Cadinol $<E p i-\alpha->$ & 12,437 & 1629 & 1638 & 2,21 \\
\hline Muurolol $<E p i-\alpha>$ & 12,602 & 1643 & 1640 & 1,58 \\
\hline Hemiterpenos oxigenados & & & & 1,03 \\
\hline Monoterpenos hidrocarbonados & & & & 0,8 \\
\hline Sesquiterpenos hidrocarbonados & & & & 81,13 \\
\hline Sesquiterpenos oxigenados & & & & 15,67 \\
\hline Total identificado & & & & 98,2 \\
\hline
\end{tabular}

RT: Tempo de retenção; $\mathbf{R}_{\text {exp }}$ : Índice de retenção determinado em relação ao $n$-alcanos $\left(C_{8}-C_{31}\right) ; \mathbf{R}_{\text {lit }}$ : Índice de retenção da literatura ${ }^{14}$; RA\%: Área relativa (área relativa do pico em relação à área total do pico no cromatograma de CG-EM) do óleo essencial das flores de Cochlospermum regium.

A atividade antioxidante foi satisfatória em todas as concentrações avaliadas, em especial para as concentrações entre 50 a $2 \mathrm{mg} \mathrm{mL}^{-1}$ onde apresentaram $100 \%$ de redução do radical livre DPPH. Neste estudo, foi observado 5 grupos, onde entre eles apresentaram diferença significativa pelo teste de Tukey $(p \leq 0,05)$, exceto para a concentração $0,062 \mathrm{mg} \mathrm{mL}^{-1}$ que não apresentou diferença significância. Na concentração de $0,25 \mathrm{mg} \mathrm{mL}^{-1}$ apresentou-se redução de $51,83 \%$ expressos em $\left(\mathrm{mg} \mathrm{mL}^{-1}\right)$, mesmo em baixas concentrações de óleo essencial diluído, observa-se importante atividade de redução de DPPH (Tabela 2). Podese inferir que os compostos cariofileno $E, \quad y$ muuroleno e biciclogermacreno pelas altas 
porcentagens obtidas no ensaio por CG-EM (Tabela 1), podem estar envolvidos na alta eficácia demonstrada a partir da atividade antioxidante para o óleo essencial das flores de $C$. regium.

Tabela 2. Avaliação da porcentagem de atividade antioxidante do óleo essencial da flor de Cochlospermum regium frente ao radical livre DPPH em diferentes concentrações $\mathrm{mg} \mathrm{mL}^{-1}$ de óleo essencial diluído.

\begin{tabular}{lc}
\hline Concentração $\mathrm{mg} \mathrm{mL}^{-\mathbf{1}}$ & $\begin{array}{c}\text { Atividade Antioxidante (\%) } \\
\text { OE }\end{array}$ \\
\hline $\mathbf{5 0}$ & $100 \pm 0,00^{\mathrm{a}}$ \\
$\mathbf{4 0}$ & $100 \pm 0,00^{\mathrm{a}}$ \\
$\mathbf{3 0}$ & $100 \pm 0,00^{\mathrm{a}}$ \\
$\mathbf{2 0}$ & $100 \pm 0,00^{\mathrm{a}}$ \\
$\mathbf{1 0}$ & $100 \pm 0,00^{\mathrm{a}}$ \\
$\mathbf{8}$ & $100 \pm 0,00^{\mathrm{a}}$ \\
$\mathbf{6}$ & $100 \pm 0,00^{\mathrm{a}}$ \\
$\mathbf{4}$ & $100 \pm 0,00^{\mathrm{a}}$ \\
$\mathbf{2}$ & $100 \pm 0,00^{\mathrm{a}}$ \\
$\mathbf{1}$ & $85,77 \pm 0,09^{\mathrm{b}}$ \\
$\mathbf{0 , 5}$ & $65,01 \pm 0,03^{\mathrm{c}}$ \\
$\mathbf{0 , 2 5}$ & $51,83 \pm 0,05^{\mathrm{c}}$ \\
$\mathbf{0 , 1 2 5}$ & $37,22 \pm 0,11^{\mathrm{d}}$ \\
$\mathbf{0 , 0 6 2}$ & $20,31 \pm 0,07^{\mathrm{ed}}$ \\
$\mathbf{0 , 0 3 1}$ & $13,18 \pm 0,05^{\mathrm{e}}$ \\
\hline
\end{tabular}

OE: Óleo Essencial flores Cochlospermum regium. Resultados referem à média de três repetições seguidos por ( \pm ) desvio padrão. Os resultados na coluna seguida de letras diferentes apresentam diferença significativa $(p \leq 0,05)$ pelo teste de Tukey.

A atividade antioxidante do óleo essencial de Prangos ferulacea foi avaliada por Bazdar et al. (2018), onde obtiveram resultado de $23,90 \% \quad\left(\mathrm{mg} \mathrm{mL}{ }^{-1}\right)$. Atividade antioxidante também foi observada em óleo essencial das flores de Oliveria decumbens avaliado por Esmaeli et al. (2018), onde obtiveram resultado de $86,1 \%\left(\mathrm{mg} \mathrm{mL}^{-1}\right)$. O efeito antioxidante está ligado diretamente pelo sinergismo entre os compostos no óleo in natura, como para os compostos isolados. Guimarães et al. (2011) discute sobre o composto citral isolado que apresenta alta eficiência como agente antioxidante a partir do modelo de DPPH; 1,8 cineol (CALDAS et al., 2020), $\alpha$-pineno, $\beta$-pineno, $O$-cimeno, D-limoneno também apresentam importante atividade antioxidante (MOOSAVY et al., 2017; OLIVEIRA et al., 2016), $\beta$-felandreno, linalol e $\beta$-cariofileno (TRAVASSOS et al., 2019), $\alpha$-terpineno e $\gamma$-terpineno (LEOMARA ANDRADE et al., 2018), timol e carvacrol (TOHIDI et al., 2017), e eugenol (XAVIER et al., 2016).

De acordo com Silva et al. (2018a), a atividade antioxidante de hidrocarbonetos sesquiterpênicos é baixa, sendo os sesquiterpenos oxigenados portadores de maior atividade, entretanto, essa afirmação não condiz com os resultados desse estudo, onde a quantidade de sesquiterpenos hidrogenados foi superior a de oxigenados (Tabela 1), apresentando alta atividade de redução de DPPH.

Observando-se a Figura 1, verifica-se que o óleo essencial das flores de $C$. regium apresentou em todas as concentrações inibição de crescimento micelial para $O$ isolado $S$. sclerotiorum. Em especial, nas concentrações de 100,50 e $25 \mu \mathrm{L} \mathrm{mL}^{-1}$ com os maiores efeitos de PIC de 79; 43 e 33\%, entretanto, as concentrações 50 e $25 \mu \mathrm{L} \mathrm{mL}^{-1}$ não apresentaram diferença significativa pelo teste $F$. Todas as concentrações diferiram significativamente, quando comparado ao fungicida de referência Frowncide $500 \mathrm{SC}$ na dose de $10 \mu \mathrm{L} \mathrm{mL}^{-1}$ com $100 \%$ de PIC. A concentração de $100 \mu \mathrm{L} \mathrm{mL}^{-1}$ demonstrou ser mais eficaz como agente fungicida biológico para o óleo essencial das flores de $C$. regium. 
Figura 1. Porcentagem de inibição de crescimento micelial de $S$. sclerotiorum em função do efeito das concentrações de óleo essencial das flores de $C$. regium. Letras iguais não diferem estatisticamente pelo teste $F$.

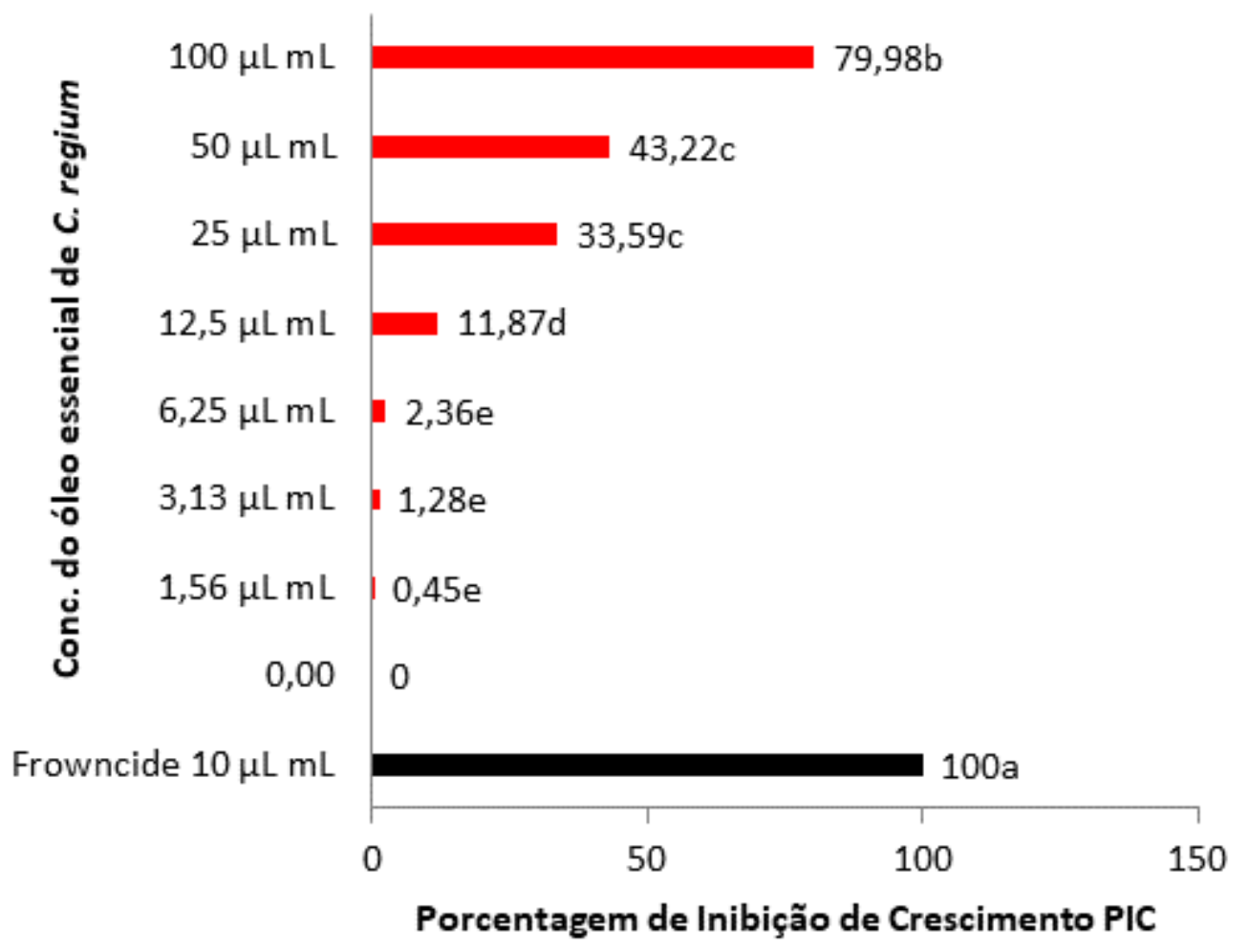

Menezes Filho et al. (2020a), encontraram excepcional atividade fungistática para os óleos essenciais dos ramos e folhas de Schinus molle com PIC de 86,41 e de $81,34 \%$, respectivamente para 0 isolado de $S$. sclerotiorum. Menezes Filho et al. (2020b), avaliaram os óleos essenciais das raízes e folhas de $C$. regium sobre o isolado de $S$. sclerotiorum onde encontraram PIC igual a 14,2 e de $60 \%$, respectivamente. Silva et al. (2018b), encontraram resultado de $94,9 \%$ de inibição de crescimento micelial para $S$. sclerotiorum em uma concentração de $300 \mu \mathrm{L} \mathrm{mL}^{-1}$, seguido de $90,8 \mathrm{e}$ $77,5 \%$ para concentrações de 200 e $100 \mu \mathrm{L} \mathrm{mL}^{-1}$, avaliando o óleo essencial das folhas de Psidium guajava. Xavier et al. (2016), avaliaram o óleo essencial das folhas de Cardiopetalum calophyllum onde obtiveram maior inibição de crescimento micelial para a concentração de 300 $\mu \mathrm{L} \mathrm{mL}^{-1}$ com $87,63 \%$ de PIC.

Quando comparados a outros estudos com óleo essencial e extratos vegetais sobre inúmeras espécies fúngicas, Jaramillo-Salazar et al. (2019), avaliaram os extratos de Anacardium occidentale sobre isolados de Aspergillus niger e Trichophyton rubrum onde apresentaram potencial antifúngico equiparável ao antifúngico de referência Anfotericina $B^{\circledR}$. Wuaden et al. (2018), encontraram importante atividade antifúngica para o óleo essencial de manjericão (Ocimum basilicum) sobre o isolado de Botrytis cinerea com boa atividade de PIC. Andrade et al. (2018), avaliaram o extrato etanólico de Byrsonima crassiflora sobre isolados de Fusarium solani e S. sclerotiorum, onde obtiveram PIC de $38 \%$ para $F$. solani em concentrações variando entre 800 a $1600 \mu \mathrm{g} 100 \mathrm{~mL}^{-1}$, e entre 19 a $37,5 \%$ para $S$. sclerotiorum em concentrações variando de 2000 a $2400 \mu \mathrm{g} 100 \mathrm{~mL}^{-1}$. Navarro et al. (2015), encontraram importante atividade fungicida para extratos vegetais das espécies de Piperaceaes (Piper umbellatum, Piper glanduligerum, Piper acuminata, Piper pesaresanum, Piper crassinervium e Piper eripodon) sobre isolados de F. solani e Fusarium oxysporum.

Os óleos essenciais extraídos de espécies vegetais empregados no controle de 
fitopatógenos apresentam grande potencialidade como agente fungicida natural, despertando o interesse para novas pesquisas na linha de produtos naturais com emprego nas áreas agrícola, farmacêutica e alimentícia. Diante do contexto, o óleo essencial das flores de $C$. regium apresentou importantes resultados, com uma rica composição química, excepcional atividade antioxidante e eficiênte atividade antifúngica sobre o isolado de $S$. sclerotiorum.

\section{Conclusões}

O óleo essencial das flores de $C$. regium apresentou baixo rendimento, em contrapartida, demonstrou ser rico em compostos pela análise cromatográfica CG-EM, bem como, apresentou atividade antioxidante, mesmo em concentrações muito baixas. Como agente antifúngico, o óleo essencial apresentou ser uma boa opção, demonstrando ser um forte candidato para novas pesquisas, na produção de fármacos e na incorporação em formulados alimentícios com atividade antioxidante, bem como potencial agente fungicida no controle de S. sclerotiorum.

\section{Agradecimentos}

Ao Instituto Federal de Educação, Ciência e Tecnologia Goiano; aos laboratórios de Química Tecnológica, Química de Produtos Naturais, Biomoléculas e Bioensaios e de Ecofisiologia; ao Herbário do IF Goiano; a Universidade Federal de São Carlos, SP, Brasil; aos órgãos de fomento CAPES, FAPEG, FINEP e CNPq pelas bolsas de mestrado para o primeiro (Antonio) e segundo autor (Wendel), e de doutorado para a terceira autora (Marcela).

\section{Referências}

ADAMS, R. P. Identification of essential oil components by gas chromatography/mass spectrometry. 4. ed. Carol Stream: Allured, 2007. p. 804.

ANDRADE, M. A.; CARDOSO, M. D. A. S. G.; BATISTA, L. R.; MALLET, A. C. T.; MACHADO, S. M. F. Essential oils of Cinnamomum zeylanicum, Cymbopogon nardus and Zingiber officinale: composition, antioxidant and Antibacterial activities. Revista Ciência Agronômica, v.43, n.2, p.399-408,

2012.

http://dx.doi.org/10.1590/S1806$\underline{66902012000200025}$
ANDRADE, L. S.; SANTOS, D. B.; CASTRO, D. B.; GUILLO, L. A.; CHEN-CHEN, L. Absence of antimutagenicity of Cochlospermum regium (Mart. and Schr.) Pilger 1924 by micronucleus test in mice. Brazilian Journal Biology, v.68, n.1, p.155-59, 2008.

http://dx.doi.org/10.1590/S1519$\underline{69842008000100022}$

ANDRADE, B. S.; MATIAS, R.; CORRÊA, B. O.; OLIVEIRA, A. K. M.; GUIDOLIN, D. G. F.; ROEL, A. R. Phytochemistry, antioxidant potential and antifungal of Byrsonima crassiflora on soil phytopathogen control. Brazilian Journal of Biology, v.78, n.1, p.140-146, 2018. http://dx.doi.org/10.1590/1519-6984.166532

BARBOSA, C. S.; PEREIRA, R. F.; FORTUNA, J. L. Antifungal activity of the essential oil of Lippia alba (Mill.) N. E. Brown (Verbenaceae) against Candida albicans. Revista Biociência, v.23, n.1, p.53-60, 2017.

BATALHA, M. A. O Cerrado não é um bioma. Biota Neotropica, v.11, n.1, p.21-24, 2011. https://doi.org/10.1590/S1676$\underline{06032011000100001}$

BATISTA, P. F.; COSTA, A. C.; MEGGUER, C. A.; LIMAS, J. S.; SALES, J. F. Overcoming dormancy and characterization of germination in Cerrado 'algodãozinho' seeds treated with dimethyl sulphoxide. South African Journal of Botany, v.92, p.89-93, 2014. https://doi.org/10.1016/i.sajb.2014.02.004

BATISTA, L. T.; SARRAZIN, S. L. F.; DE MOURA, V. M.; SANTOS, I. G. C.; DUVOISIN JÚNIOR, S.; ALBUQUERQUE, P. M. Composição química, atividade antimicrobiana e antioxidante do óleo essencial de Aniba parviflora (Meisn) Mez. Revista Fitos, v.13, n.3, p.181-191, 2019. https://doi.org/10.32712/2446-4775.2019.788

BAZDAR, M.; SADEGHI, H.; HOSSEINI, S. Evaluation of oil profiles, total phenols and phenolic compounds in Prangos ferulacea leaves and flowers and their effects on antioxidant activities. Biocatalysis and Agricultural Biotechnology, v.14, p.418-23, 2018. https://doi.org/10.1016/i.bcab.2018.04.009

BEUCHLE, R.; GRECCHI, R. C.; SHIMABUKURO, Y. E.; SELIGER, R.; EVA, H. D.; SANO, E.; ACHARD, F. 
Land cover changes in the Brazilian Cerrado and Caatinga biomes from 1990 to 2010 based on a systematic remote sensing Sampling approach. Applied Geography, v.58, p.116-127, 2015. https://doi.org/10.1016/i.apgeog.2015.01.017

BEZERRA, J. W. A.; RODRIGUES, F. C.; COSTA, A. R.; LEMOS, S. M. A.; PEREIRA, M. L. A.; RODRIGUES, A. S. Avaliação da ação inseticida e larvicida do óleo essencial de Tarenaya spinosa (Jacq.) Raf. (mussambê) (Cleomaceae). Revista Cubana de Plantas Medicinales, v.24, n.2, p.e814, 2019. http://revplantasmedicinales.sld.cu/index.php/pl a/article/view/814/375

BOMFIM, N. S.; NAKASSUGI, L. P.; OLIVEIRA, J. F. P.; KOHIYAMA, C. Y.; MOSSINI, S. A. G.; GRESPAN, R.; NERILO, S. B.; MALLMAN, C. A.; ABREU FILHO, B. A.; MACHINSKI JÚNIOR, M. Antifungal activity and Inhibition of fumonisin production by Rosmarinus officinalis L. essential oil in Fusarium verticillioides (Sacc.) Nirenberg. Food Chemistry, v.166, n.1, p.330-336, 2015. https://doi.org/10.1016/i.foodchem.2014.06.019

BRUM, R. L.; HONDA, N. K.; HESS, S. C.; CRUZ, A. B.; MORETTO, E. Antibacterial activity of Cochlospermum regium essential oil. Fitoterapia, v.68, n.1, p.79, 1997.

CALDAS, G. F. R.; DANTAS, L. P.; CAMURÇA, A. J. S.; TEIXEIRA, M. M. S.; RODRIGUES, F. F. G.; DA COSTA, J. G. M.; WANDERLEY, A. G. Propriedades farmacológicas e toxicologia do óleo essencial de Hyptis martiusii Benth. (cidreira-brava) e de seu composto majoritário 1,8-cineol: uma revisão. Revista Interfaces, v.8, n.1, p.461-471, 2020. https://doi.org/10.16891/2317

434X.v8.e1.a2020.pp461471

CAMILLO, J.; SCHERWINSKI-PEREIRA, J. E.; VIEIRA, R. F.; PEIXOTO, J. R. In vitro Conservation of Cochlospermum regium (Schrank) Pilg.Cochlospermaceae under minimal growth storage. Revista Brasileira de Plantas Medicinais, v.11, n.2, p.184-89, 2009.

CAMILLO, J.; MARTINS, R. C.; NETO, G. G.; GULIAS, A. P. S. M.; FILGUEIRAS, A. D.; COSTA, L. C.; SILVA, D. B. Cochlospermum regium (Algodãodo-campo). Espécies Nativas da Flora Brasileira de Valor Econômico Atual ou Potencial: Plantas para o futuro - Região Centro-Oeste. Brasília:
Ministério do Meio Ambiente, 2016. Cap. 5, p. 717-30.

CARNEIRO, M. S.; SILVEIRA, A. P.; SANTOS GOMES, V. Comunidade rural e escolar na valorização do conhecimento sobre plantas medicinais. Biotemas, v.29, n.2, p.89-99, 2016. https://doi.org/10.5007/2175-

\section{$\underline{7925.2016 v 29 \mathrm{n} 2 \mathrm{p} 89}$}

CERQUEIRA, M. D.; MARQUES, E. E. J.; MARTINS, D.; ROQUE, N. F.; CRUZ, F. G.; GUEDES, M. L. D. A. $S$. Seasonal variation of the composition of essential oil from Myrcia salzmannii Berg. (Myrtaceae). Química Nova, v.32, n.6, p.1544-48, $2009 . \quad$ http://dx.doi.org/10.1590/S010040422009000600035

COSTA, A. R.; PEREIRA, P. S.; BARROS, L. M.; DUARTE, A. E.; GOMEZ, M. C. V.; ROLÓN, M.; VIDAL, C. A. S.; MAIA, A. J.; BRAGA, M. F. B. M.; COUTINHO, H. D. A citotoxicidade e a avaliação da atividade antiprotozoária de Melissa officinalis L (cidro-melisa). Revista Cubana de Plantas Medicinales, v.21, n.4, 2016. http://revplantasmedicinales.sld.cu/index.php/pl a/article/view/380/201

ESMAELI, H.; KARAMI, A.; MAGGI, F. Essential oil composition, total phenolic and flavonoids contents, and antioxidant activity of Oliveria decumbens Vent. (Apiaceae) at different phenological stages. Journal of Cleaner Production, v.198, p.91-95, 2018. https://doi.org/10.1016/i.jclepro.2018.07.029

GARCIA, R. Á.; JULIATTI, F. C.; BARBOSA, K. A. G.; CASSEMIRO, T. A. Atividade antifúngica de óleo e extratos vegetais sobre Sclerotinia sclerotiorum. Bioscience Journal, v.28, n.1, p.48-57, 2012. http://www.seer.ufu.br/index.php/biosciencejou rnal/article/view/8174

GASTALDI, B.; ASSEF, Y.; VAN BAREN, C.; LIRA, P. DI L.; RETTA, D.; BANDONI, A. L.; GONZÁLEZ, S. B. Actividad antioxidante en infusiones, tinturas y aceites esenciales de especies nativas de la Patagonia Argentina. Revista Cubana de Plantas Medicinales, v. 21, n. 1, 2016. http://revplantasmedicinales.sld.cu/index.php/pl a/article/view/306/157

GUIMARÃES, L. G. L.; CARDOSO, M. G.; DE SOUSA, P. E.; DE ANDRADE, J.; VIEIRA, S. S. 
Atividades antioxidante e fungitóxica do óleo essencial de capim-limão e do citral. Revista Ciência Agronômica, v. 42, n. 2, p. 464-472, 2011. https://doi.org/10.1590/S1806-

$\underline{66902011000200028}$

HAMID, M. R.; XIE, J.; WU, S.; MARIA, S. K.; ZHENG, D.; HAMIDOU, A. A.; WANG, Q.; CHENG, J.; FU, Y.; JIANG, D. A novel Deltaflexivirus that infects the plant fungal pathogens, Sclerotinia sclerotiorum, can be transmitted among host vegetative incompatible strains. Viruses, v.10, n.6, p.295, 2018. https://doi.org/10.3390/v10060295

JUIZ, P. J. L.; DA SILVA, F.; CAMPOS, M. J. A.; UETANABARO, A. P. T.; ALVES, R. J. C.; LUCCHESE, A. M. Antimicrobial activity of essential oil of Ocimum americanum and Ocimum basilicum against periodontopathogens. Brazilian Journal Periodontology, v. 26, n. 4, p. 7-14, 2016.

JARAMILLO-SALAZAR, M. T.; OCAMPO-SERNA, D. M.; CRUZ-NARANJO, B. D.; GALVIS-GARCÍA, J. H. Actividad antibacteriana y antifúngica de los extractos de diferente polaridad de Anacardium occidentale. Revista Cubana de Plantas Medicinales, v. 24, n. 2, p. e677, 2019. http://revplantasmedicinales.sld.cu/index.php/pl a/article/view/677/381

JULIATTI, F. C.; CRATO, F. F.; JULIATTI, F. C.; COUTO, K. R.; JULIATTI, B. C. M. Escala diagramática para avaliação da severidade de mofo branco em soja. Bioscience Journal, v.29, n.3, p.676-680, 2013. http://www.seer.ufu.br/index.php/biosciencejou rnal/article/view/22440

KUMAR, V. MATHELA, C. S.; TEWARI, G.; SINGH, D.; TEWARI, A. K.; BISHT, K. S. Chemical composition and antifungal activity of essential oils from three Himalayan erigeron species. LWT - Food Science and Technology, v.56, n.2, p.278283, 2014. https://doi.org/10.1016/i.lwt.2013.12.007

MARCO, C. A.; SANTOS, H. R.; SOUSA FEITOSA, A. G.; FEITOSA, J. V.; COSTA, J. G. M. Teor, rendimento e qualidade do óleo essencial de Vanillosmopsis arborea (Gardner) Baker (candeeiro) e sua ação alelopática. Revista Cubana de Plantas Medicinales, v.20, n.1, 2015. http://revplantasmedicinales.sld.cu/index.php/pl a/article/view/115/118

MENDONÇA, R. C.; FELFILI, J, M.; WALTER, B. M. T.; SILVA JÚNIOR, M. C.; REZENDE, A. V.; FILGUEIRAS, T. S.; NOGUEIRA, P. E. Flora vascular do cerrado. Pp. 287-556. In: SANO, S. M.; ALMEIDA, S. P. (Eds.). Cerrado: ambiente e flora. Planaltina: Embrapa Cerrados, 1998.

MENEZES FILHO, A. C. P.; SOUSA, W. C.; CASTRO, C. F. S. Composição química dos óleos essenciais de Schinus molle e atividade antifúngica em Sclerotinia sclerotiorum. Colloquium Agrariae, v.16, n.3, p.115-123, 2020a. http://revistas.unoeste.br/index.php/ca/article/vi ew/3078

MENEZES FILHO, A. C. P.; DE SOUSA, W. C.; CASTRO, C. F. S. Atividades antioxidante e antifúngica dos óleos essenciais de Cochlospermum regium frente à Sclerotinia sclerotiorum e Colletotrichum gloeosporioides. Colloquium Agrariae, v. 16, n. 1, p. 109-116, 2020b.

https://doi.org/10.5747/ca.2020.v16.n1.a353

MENEZES FILHO, A. C. P. Avaliação química, antifúngica e antioxidante do óleo essencial da flor de Fridericia platyphylla (Cham.) L. G. Lohmann. Scientia Naturalis, v.2, n.1, p 42-58, 2020b.

https://revistas.ufac.br/index.php/SciNat/article/ view/3285

MENEZES FILHO, A. C. P.; CASTRO, C. F. S. Avaliação dos extratos vegetais de Cochlospermum regium em um Cerrado ralo. Global Science and Technology, v. 13, n. 1, p. 117, 2020.

MEZZA, G. N.; BORGARELLO, A. V.; GROSSO, N. R.; FERNANDEZ, H.; PRAMPARO, M. C.; GAYOL, M. F. Antioxidant activity of rosemary essential oil fractions obtained by molecular distillation and their effect on oxidative stability of sunflower oil. Food Chemistry, v.242, n.1, p.9-15, 2018. https://doi.org/10.1016/j.foodchem.2017.09.042

MOOSAVY, M. H.; HASSANZADEH, P.; MOHAMMADZADEH, E.; MAHMOUDI, R.; KHATIBI, S. A.; MARDANI, K. Antioxidant and antimicrobial activities of essential oil of lemon (Citrus limon) peel in vitro and in a food model. 
Journal of Food Quality \& Hazards Control, v.4, p.42-48, 2017. http://ifahc.ssu.ac.ir/article-1-335en.html

MORAIS, S. M.; JÚNIOR, F. E. A. C.; SILVA, A. R. A.; NETO, J. S. M. Atividade antioxidante de óleos essenciais de espécies de Croton do nordeste do Brasil. Química Nova, v.29, n.5, p.907-10, 2006. http://dx.doi.org/10.1590/S0100$\underline{40422006000500004}$

NAVARRO, Y. M. C.; GARCÍA, L. R. P.; MOSQUERA, O. M. Actividad antioxidante y antifúngica de Piperaceaes de la flora colombiana. Revista Cubana de Plantas Medicinales, v.20, n.2, 2015. http://revplantasmedicinales.sld.cu/index.php/pl a/article/view/125/122

NASCIMENTO, M. N. G.; JUNQUEIRA, J. G. M.; TEREZAN, A. P.; SEVERINO, R. P.; SILVA, T. S.; MARTINS, C. H. G.; SEVERINO, V. G. P. Chemical composition and antimicrobial activity of essential oils from Xylopia aromatica (Annonaceae) flowers and leaves. Revista Virtual de Química, v.10, n.5, p.1578-1590, 2018. http://dx.doi.org/10.21577/1984-6835.20180107

NETO, G. G.; MORAIS, R. G. Recursos medicinais de espécies do Cerrado de Mato Grosso: um estudo bibliográfico. Acta Botanica Brasílica, v.17, n.4, p.561-584, 2003. https://doi.org/10.1590/S0102-

$\underline{33062003000400009}$

OLIVEIRA, J. D.; ALVES, C. C. F.; MIRANDA, M. L. D.; MARTINS, C. H. G.; SILVA, T. S.; AMBROSIO, M. A. L. V.; ALVES, J. M.; SILVA, J. P. Rendimento, composição química e atividades antimicrobiana e antioxidante do óleo essencial de folhas de Campomanesia adamantium submetidas a diferentes métodos de secagem. Revista Brasileira de Plantas Medicinais, v.18, n.2, p.502510, 2016. https://doi.org/10.1590/1983$\underline{084 X / 15 \quad 206}$

ROUQUAYROL, M. Z.; FONTELES, M. C.; ALENCAR, J. E.; ABREU-MATOS, F. J. D.; CRAVEIRO, A. A. Atividade moluscicida de óleos essenciais de plantas do nordeste brasileiro. Revista Brasileira de Pesquisa Médica Biológica, v.13, n.4-6, p.13543, 1980.

SILVA, Y. S.; SILVA, E. N.; SILVA, D. M.; DIAS CARNEIRO, M. V.; RIBEIRO, W. S. Seletividade de extratos naturais a pragas, inimigos naturais, parasitoides e predadores em sistema de produção agroecológico vegetal. Caderno Verde de Agroecologia e Desenvolvimento Sustentável, v.9, n.7, e-7086, 2019c. https://doi.org/10.18378/cvads.v9i7.7086

SILVA, L. A.; RAPOSO, J. D. A.; CAMPOS, L. P. G.; CONCEIÇÃO, E. C.; OLIVEIRA, R. B.; MOURÃO, R. $\mathrm{H}$. V. Atividade antioxidante do óleo essencial de Myrcia sylvatica (G. Mey.) DC. por diferentes métodos de análises antioxidantes (ABTS, DPPH, FRAP, $\beta$-caroteno/ácido linoleico). Revista Fitos, v.12, n.2, p.117-126, 2018a. https://doi.org/10.5935/2446-4775.20180011

SILVA, E. A. J.; DA SILVA, V. P.; ALVES, C. C. F.; ALVES, J. M.; SOUCHIE, E. L.; BARBOSA, L. C. A. Chemical composition of the essential oil of Psidium guajava leaves and its toxicity against Sclerotinia sclerotiorum. Semina: Ciências Agrárias, v.39, n.2, p.865-874, 2018b. https://doi.org/10.5433/1679-

$\underline{0359.2018 v 39 n 2 p 865}$

SILVA, E. R.; PAWLOWSKI, Â.; LAZAROTTO, D. C.; SOARES, G. L. G. Antioxidant activity of essential oils from leaves of Schinus lentiscifolius Marchand. Revista Cubana de Plantas Medicinales, v.24, n.2, p.e783, 2019a. http://revplantasmedicinales.sld.cu/index.php/pl a/article/view/783/382

SILVA, T. K. R.; HITZ, D.; MAIA, A. J.; NOVELLO, D.; SCHWARZ, K.; JARDINETTI, V. A. Use of Cymbopogon citratus essential oils for preservation of Fragaria ananassa after conventional harvesting. Revista Cubana de Plantas Medicinales, v.24, n.2, p.e650, 2019b.

SILA, A. F.; RABELO, M. F. R.; ENOQUE, M. M. Diversidade de angiospermas e espécies medicinais de uma área de Cerrado. Revista Brasileira de Plantas Medicinais, v.17, n.4, supl. III, p.1016-1030, 2015.

SÓLON, S.; CAROLLO, C. A.; BRANDÃO, L. G.; DE MACEDO, C. D. O. S. S.; KLEIN, A.; DIAS-JÚNIOR, C. A.; DE SIQUEIRA, J. M. Phenolic derivatives and other chemical compounds from Cochlospermum regium. Química Nova, v.35, n.6, p.1169-72, $2012 . \quad \quad$ http://dx.doi.org/10.1590/S0100$\underline{40422012000600019}$ 
SÓLON, S.; BRANDÃO, L. F. G.; DE SIQUEIRA, J. M. Genus Cochlospermum Kunth with emphasis on ethnobotanic, pharmacological, toxicological, and chemical aspects of the Cochlospermum regium (Mart. et Schr.) Pilger. Revista Eletrônica de Farmácia, v.4, n.3, p.1-22, 2009. https://doi.org/10.5216/ref.v6i3.7649

SOUHAIEL, N.; SIFAOUI, I.; HASSINE, D. B.; BLETON. J.; BONOSE, M.; MOUSSA, F.; PIÑERO, J. E.; LORENZO-MORALES, J.; ABDERRABBA, $M$. Ammoides pusilla (Apiaceae) essential oil: acitivity against Acanthamoeba castellanii Neff. Experimental Parasitology, v.183, p.99-103, 2017.

https://doi.org/10.1016/i.exppara.2017.10.011

SOUSA, R. F.; SOUSA, J. A. Metabólicos secundários associados a estresse hídrico e suas funções nos tecidos vegetais. Revista Brasileira de Gestão Ambiental, v.11, n.01, p.01-08, 2017. https://editoraverde.org/gvaa.com.br/revista/ind ex.php/RBGA/article/view/5008

TOHIDI, B.; RAHIMMALEK, M.; ARZANI, A. Essential oil composition, total phenolic, flavonoid contents, and antioxidant activity of Thymus species collected from different regions of Iran. Food Chemistry, v.220, p.153-161, 2017. https://doi.org/10.1016/i.foodchem.2016.09.203

XAVIER, M. N.; ALVES, J. M.; CARNEIRO, N. S.; SOUCHIE, E. L.; SILVA, E. A. J.; MARTINS, C. H. G.; AMBROSIO, M. A. L. V.; EGEA, M. B.; ALVES, C. C. F.; MIRANDA, M. L. D. Composição química do óleo essencial de Cardiopetalum calophyllum Schltdl. (Annonaceae) e suas atividades antioxidante, antibacteriana e antifúngica. Revista Virtual de Química, v.8, n.5, 2016. http://dx.doi.org/10.21577/1984-6835.20160101

WUADEN, C. R.; GAIO, I.; SPERHACKE, T.; BARRO, J. P.; MILANESI, P. M. Atividade antifúngica do extrato alcoólico de própolis, álcool de cereais e do óleo essencial de manjericão sobre Botrytis cinerea. Colloquium Agrariae, v.14, n.2, p.48-55, 2018.

https://doi.org/10.5747/ca.2018.v14.n2.a205 